\title{
Chain Properties of Rule Closures
}

\author{
Miki Hermann \\ Centre de Recherche en Informatique de Nancy, CNRS and INRIA-Lorraine, Campus \\ Scientifique, BP 239, 54506 Vandauvre-lès-Nancy, France
}

Keywords: Term rewriting systems; Knuth-Bendix completion procedure; Divergence

\begin{abstract}
This article introduces a generalisation of the crossed rule approach to the detection of Knuth-Bendix completion procedure divergence. It introduces closure chains, which are special rule closures constructed by means of particular substitution operations and operators, as a suitable formalism for progress in this direction. Supporting substitution algebra is developed first, followed by considerations concerning rule closures in general, concluding with an investigation of closure chain properties. Issues concerning the narrowing process are not discussed here.
\end{abstract}

\section{Introduction}

In [HeP86] the importance of forward closures and later also backward closures for the definition of crossed rules was discovered. During the attempts to generalise the crossed rule notion it became apparent that their construction is strictly dependent on special forward or backward rule closures, called forward/backward chains. These closure chains are derived from rule closures by substitution variable constraints, and are constructed by means of special substitution operations and iterative operators. It is this iterative construction of the closure chains that makes them most suitable for the description of the Knuth-Bendix procedure divergence. Therefore attention is focused on a sufficiently general definition with the potential to describe the largest class of possible cases by their structural entities, followed by a deep investigation of their properties. Rather than offering a plethora of different isolated types of objects on the same level (although in examples the differences are strictly

Correspondence and offprint requests to: Miki Hermann. Centre de Recherche en Informatique de Nancy, CNRS and INRIA-Lorraine, Campus Scientifique, BP 239, 54506 Vandœuvre-lès-Nancy, France. 
pointed out), a synthetic approach has been applied, yielding a unifying view with a family of definitions interconnected by a duality principle.

The article is divided into four sections. Section 2 describes the motivation for investigations of closure chain properties on the basis of two divergence examples. Section 3 introduces the basic notation in term rewriting systems theory and provides the rule closure definitions - the main object of the article. Section 4 presents a special substitution algebra. In its first part it contains the definitions of two substitution operations - sum and join - with appropriate propositions proving their necessary properties. Upon the sum operation three iterative substitution operators - exponent, W- and T-operator - have been built and their properties have been proved in the second part of Section 4 . Section 5 investigates the properties of rule closures. Its first part focuses on the structure and construction of rule closures in general, presenting a closure set generating procedure, and proving the closure set finiteness undecidability. The second part is introduced by general considerations concerning the construction of closure chains, followed by their definition. Section 5 is concluded by characterisation theorems of closure sets when they contain a closure chain, and by exact constructions of closure chains, taking advantage of the iterative operators from Section 4. An index containing all newly defined symbols in the paper is appended.

\section{Motivation}

Applying the Knuth-Bendix procedure to complete a given term rewriting system may result in the canonical term rewriting system, if it halts, or in a diverging process trying to generate an infinite set of rewrite rules. The first result concerning structural properties of an infinite set of rules generated by the Knuth-Bendix completion procedure is due to Huet [Hue81]. Further attempts to study the reasons of Knuth-Bendix procedure divergence through investigation of the structural properties of the generated rules were undertaken in [HeP86]. The notion of crossed rules as a sufficient criterion for proving divergence of the Knuth-Bendix procedure emerged from those investigations. Recently, complementary research by Kirchner [Kir89] focused on the description of infinite sets of rules generated by diverging KnuthBendix procedure by finite means, developing the notion of meta-rules and related objects.

The study of divergence patterns emerged from the observation of natural rewrite systems with diverging performance. Trying to enlarge the definition of crossed rules from [HeP86] so that it would cover a more general class of rewrite systems whose structural properties imply the divergence process appealed to the necessity of more insight into the behaviour of certain rewrite rule sequences.

Example 2.1. A natural example of a diverging rewrite system is associativity and endomorphism, studied in another context in [BeL87].

$$
\begin{aligned}
(x * y) * z & \rightarrow x *(y * z) \\
f(x) * f(y) & \rightarrow f(x * y)
\end{aligned}
$$

If we try to complete this system, choosing the operator precedence $*>f$ with the left-to-right status of $*$, we get a diverging process. 
A more general, but rather artificial, example is the rewrite system ${ }^{1}$

$$
\begin{aligned}
d(x \oplus h(y)) & \rightarrow y \\
(x \otimes y) \oplus y & \rightarrow k(x \oplus k(y))
\end{aligned}
$$

If we try to complete this rewrite system in RevE [Les83], choosing the operator precedence ${ }^{2} \otimes>\oplus$ and $\otimes>k$, the process diverges while generating the infinite rule family

$$
d\left(k^{n}\left(x \oplus k^{n}(h(y))\right)\right) \rightarrow y
$$

The second rewrite rule (1) in the previous example can always be superposed with a just-generated rule, producing a further critical pair of terms which is in turn oriented into a new rule. When we take a closer look at this example, the divergence is made possible because there is always a subterm on the left-hand side of a just-generated rule which is an instance of the right-hand side in rewrite rule (1). Where does it come from? Because the leftand right-hand terms in rewrite rule (1) can be superposed. Let us describe the process in detail. The rewrite rule (1) has a substitution instance

$$
((x \otimes k(y)) \otimes y) \oplus y \rightarrow k((x \otimes k(y)) \oplus k(y))
$$

produced by $\varphi_{1}=[x \mapsto x \otimes k(y)]$, and another substitution instance

$$
(x \otimes k(y)) \oplus k(y) \rightarrow k(x \oplus k(k(y)))
$$

produced by $\varphi_{2}=[y \mapsto k(y)]$. Now, the left-hand side of the rule instance (3) is a subterm of the right-hand side in the rule instance (2). Let us replace this mentioned subterm by the right-hand side of the rule-instance (3), yielding the rule

$$
((x \otimes k(y)) \otimes y) \oplus y \rightarrow k(k(x \oplus k(k(y))))
$$

Now, the left-hand side term in rule (1) can be superposed with the right-hand side term in rule (4), producing a similar new rule again. This process can be iterated infinitely many times, resembling the original completion process. As described earlier in [HeP86], the iteration basis need not be only a single rule but it can be constructed as a sequence of individual rules. As a matter of fact, this infinite iteration is the basis of the previous divergence process. Therefore we will focus our attention on such infinitely chaining sequences, investigating their properties.

The rewrite system from Example 2.1 does not represent the only possible class of diverging rewrite systems.

Example 2.2. Consider the rewrite system

$$
\begin{aligned}
(x \otimes h(y)) \oplus y & \rightarrow(x \oplus y) \otimes y \\
(x \oslash y) \otimes y & \rightarrow x
\end{aligned}
$$

If we try to complete this rewrite system in REVE, choosing the operator precedence $\oplus>\otimes$ in the recursive path ordering, the process diverges while generating the infinite rule family

$$
\begin{aligned}
\left(\left(\left(\left(x \oslash h^{n+1}(y)\right) \oplus h^{n}(y)\right) \oplus \cdots \oplus h(y)\right) \oplus y\right) \otimes y & \\
& \rightarrow\left(\left(x \oplus h^{n}(y)\right) \oplus \cdots \oplus h(y)\right) \oplus y
\end{aligned}
$$

\footnotetext{
${ }^{1}$ Circled operators are to be considered only as syntactic objects, and not as real operators.

${ }^{2}$ Other possibilities for choosing precedence are available.
} 
Comparison of the previous example with 2.1 reveals the difference. While in Example 2.1 it was always the subterm on the left-hand side of the generated rule that was unifiable with the left-hand term of the rule (1), here it is the whole left-hand side of the generated rule that is unifiable with the subterm on the left-hand side of the rule (5). Further, all the critical pairs of terms generated during the completion process in 2.2 are oriented in the opposite direction, while in 2.1 the direction was retained.

This second type of divergence results from the fact that the right- and left-hand sides in rule (5) can be superposed. Comparing the superposition possibility in (1) and (5), the major term is now on the left-hand side, while in (1) it was on the right. But the chaining principle is retained, only the direction is reversed. While in (1) it was a forward chaining process, in (5) the chaining direction is backward. Also in this chaining type the iteration basis need not be only a single rule but a rule sequence.

In the following sections we develop a unifying approach to both chaining types, providing sufficient (and sufficiently general) conditions for the infinite iteration.

\section{Basic Notation and Definitions}

For completeness, the notation used in term rewriting system theory is introduced in the first part of this chapter. The second section contains the closure definitions.

\subsection{Term Rewriting Systems}

We adopt the notation of [DeJ89]. Let $\mathscr{F}$ be a finite or enumerable set of function symbols graded by arity (signature). $\mathscr{F}_{0}$ denotes the constants. Let $\mathscr{X}$ be an enumerable set of variables such that $\mathscr{F} \cap \mathscr{S}=\varnothing$. Denote by $\mathscr{T}(\mathscr{F}, \mathscr{Z})$ the set of all terms (free algebra) over variables $\mathscr{X}$ and symbols $\mathscr{F}$. $\operatorname{Var}(t)$ denotes the set of all variables in the term $t$.

Let $N^{*}$ be the set of strings of natural numbers with a special symbol $\Lambda \in N^{*}$ for the empty string and a concatenation operation on $N^{*}$. Using the elements of $N^{*}$ as labels, the terms can be viewed as labeled trees. A term $t$ is a partial function $N^{*} \rightarrow \mathscr{F} \cup \mathscr{X}$ such that its domain $\operatorname{Pos}(t)$ satisfies the following properties:

1. if $t \in \mathscr{F}_{0} \cup \mathscr{X}$ then $\operatorname{Pos}(t)=\{\Lambda\}$.

2. if $t=f\left(t_{1}, \ldots, t_{n}\right)$ then $\operatorname{Pos}(t)=\{\Lambda\} \cup\left\{i . a . \mid i=1, \ldots, n\right.$ and $\left.a \in \operatorname{Pos}\left(t_{i}\right)\right\}$.

$\operatorname{Pos}(t)$ is the set of positions of the term $t$. The subset of non-variable positions of $t$ is denoted by $\mathscr{F} \operatorname{Pos}(t)$.

A subterm of $t$ at a position $a \in P o s(t)$ is denoted by $\left.t\right|_{a}$. If $t=f\left(t_{1}, \ldots, t_{n}\right)$ then $\left.t\right|_{\Lambda}=t$ and $\left.t\right|_{i a}=\left.t_{i}\right|_{a}$ for all $i=1, \ldots, n$. Denote by $s[t]_{a}$ a new term obtained from the term $s$ after replacing its subterm $\left.s\right|_{a}$ by $t$. For properties of replacement see [Ros73].

A substitution is a function $\sigma: \mathscr{X} \rightarrow \mathscr{T}(\mathscr{F}, \mathscr{X})$ such that $x \sigma=x$ holds for all but a finite number of variables. Denote a substitution $\sigma$ by $\left[x_{1} \mapsto t_{1}, \ldots, x_{n} \mapsto\right.$ $\left.t_{n}\right]$ when the terms $t_{i}$ are substituted for the variables $x_{i}$ and $x_{i} \neq t_{i}$, for $i=1, \ldots, n$. An empty substitution is denoted by []. Substitutions have a 
homomorphic extension on the terms. Denote by $\operatorname{Dom}(\sigma), \operatorname{Ran}(\sigma)$, and $\operatorname{Var}(\sigma)$ the domain, range, and all variables of a substitution $\sigma$, respectively. A substitution $\sigma$, such that $x \sigma \in \mathscr{Z}$ and $x \sigma=y \sigma$ implies $x=y$ for all $x, y \in$ $\operatorname{Dom}(\sigma)$, is a variable renaming. Substitutions need not be idempotent in our approach.

Two terms $s$ and $t$ are unifiable if, and only if, there is an idempotent substitution $\sigma$ such that $s \sigma=t \sigma$. The substitution $\sigma$ is called a unifier. The substitution $\sigma$ is called the most general unifier (up to variable renaming) for $s$ and $t$ if for all unifiers $\varphi$ of $s$ and $t$ there exists a substitution $\psi$, such that $\varphi=\sigma \psi$. The substitution $\sigma$ on term $t$ is a substitution in own variables of $t$ if it does not introduce new variables, i.e. $\operatorname{Var}(t \sigma) \subseteq \operatorname{Var}(t)$, and does not contain a variable renaming. This notion can be enlarged to a set of substitutions.

A rewrite rule is an ordered pair of terms $r=(s, t)$ such that $\operatorname{Var}(t) \subseteq$ $\operatorname{Var}(s)$. The rule $p^{o p}=t \rightarrow s$ is the opposite rule to $p=s \rightarrow t$, if $\operatorname{Var}(s)=\operatorname{Var}(t)$. A term rewriting system (or rewrite system for short) is a finite set of rules $R=\{s \rightarrow t \mid s, t \in \mathscr{T}(\mathscr{F}, \mathscr{Z})\}$. A term rewriting system $R$, where $\operatorname{Var}(s)=$ $\operatorname{Var}(t)$ holds for all rewrite rules $s \rightarrow t \in R$, is called a variable preserving rewrite system. The term rewriting system constructed from a variable preserving rewrite system $R$ by turning the rules in the opposite direction is the set of rules $R^{o p}=\{t \rightarrow s \mid s \rightarrow t \in R\}$, called the opposite term rewriting system to $R$.

\subsection{Rule Closures}

The following constructions come from Lankford and Musser [LaM78] and also from Guttag, Kapur and Musser [GKM83]. They are mentioned also by Dershowitz [Der87].

Definition 3.3. Let $R$ be an arbitrary set of rules. The set of forward closures $F C(R)$ of $R$ is inductively defined as follows:

1. Every rule $s \rightarrow t$ from $R$ is a forward closure $s \bowtie t$.

2. Let $s_{1} \bowtie t_{1}, s_{2} \bowtie t_{2}$ be two forward closures. If $\left.t_{1}\right|_{a} \sigma=s_{2} \sigma$ holds for a most general unifier $\sigma$ and position $a \in \mathscr{F P o s}\left(t_{1}\right)$, then $s_{1} \sigma \triangleright t_{1} \sigma\left[t_{2} \sigma\right]_{a}$ is a forward closure.

The set of backward closures $B C(R)$ of $R$ is inductively defined as follows:

1. Every rule $s \rightarrow t$ from $R$ is a backward closure $s \mapsto t$.

2. Let $s_{1} \mapsto t_{1}, s_{2} \mapsto t_{2}$ be two backward closures. If $t_{1} \sigma=\left.s_{2}\right|_{a} \sigma$ holds for a most general unifier $\sigma$ and position $a \in \mathscr{F P}$ os $\left(s_{2}\right)$, then $s_{2} \sigma\left[s_{1} \sigma\right]_{a} \mapsto t_{2} \sigma$ is a backward closure.

The set of overlap closures $O C(R)$ of $R$ is inductively defined as follows:

1. Every rule $s \rightarrow t$ from $R$ is an overlap closure $s \leftrightarrow t$.

2. Let $s_{1} \leftrightarrow t_{1}, s_{2} \mapsto t_{2}$ be two overlap closures. If $\left.t_{1}\right|_{a} \sigma=s_{2} \sigma$ holds for a most general unifier $\sigma$ and position $a \in \mathscr{F} P o s\left(t_{1}\right)$, then $s_{1} \sigma \rightarrow t_{1} \sigma\left[t_{2} \sigma\right]_{a}$ is an overlap closure.

3. Let $s_{1} \mapsto t_{1}, s_{2} \mapsto t_{2}$ be two overlap closures. If $t_{1} \sigma=\left.s_{2}\right|_{a} \sigma$ holds for a most general unifier $\sigma$ and position $a \in \mathscr{F P} \operatorname{Pos}\left(s_{2}\right)$, then $s_{2} \sigma\left[s_{1} \sigma\right] \mapsto t_{2} \sigma$ is an overlap closure. 
The forward and overlap closures provide the essence of a specific method for proving termination of term rewriting systems. A survey of related results can be found in [Der87]. These notions are related to the narrowing process, as defined by Fay [Fay79], Hullot [Hul80], Lankford [Lan75], and Slagle [Sla74]; and to the superposition process defined in [GKM83].

\section{Substitution Operations and Operators}

To be able to construct specific iterations observed during the divergence of the completion process, it is necessary to develop a substitution algebra with its own operations. The substitution operations introduced appear to be necessary during the investigation of closure chain properties. Their properties build the basis for proving results in the subsequent sections.

In the first part, two substitution operations - the sum and join-are defined, and successively their properties are proved. Upon the sum operation two other substitution operators (W- and T-operator) are built. These two operators are defined as iterations of the substitution sum on different basic level substitutions. As will be shown in the following sections, the iteration properties of the operators make them most suitable for proving specific closure chain properties, and even developing the notion of a closure chain as an iterated object. The properties of these iterative substitution operators are proved at the end of this section.

\subsection{Substitution Operations}

Definition 4.4. Let $\varphi$ and $\psi$ be arbitrary substitutions.

The sum of $\varphi$ and $\psi$ is the substitution $\varphi \Delta \psi=[x \mapsto x \varphi \psi \mid x \in \operatorname{Dom}(\varphi)$, $x \varphi \psi \neq x]$.

The join of $\varphi$ and $\psi$ is the substitution $\varphi \sqcup \psi=\varphi \cup[x \mapsto x \psi \mid x \in \operatorname{Dom}(\psi)-$ $\operatorname{Dom}(\varphi)]$.

It is to be recalled that the substitution composition $\varphi \psi$ can be written as $(\varphi \Delta \psi) \cup[x \mapsto x \psi \mid x \in \operatorname{Dom}(\psi)-\operatorname{Dom}(\varphi)]$.

The substitution sum and join are well-defined (i.e. they yield a substitution). This can be easily deduced from the definition. The sum is a composition restricted to the variables of the first substitution, therefore sometimes denoted by $\left.(\varphi \psi)\right|_{\operatorname{Dom}(\varphi)}($ this notation will not be used in this article). The substitution join is an operation helpful in proving the core Lemma 4.8 of this subsection.

The substitution sum is in general neither commutative nor associative. Other properties are contained in the following lemma.

Lemma 4.5. Let $\varphi, \psi$ and $\sigma$ be substitutions.

1. The identity $\varphi \psi=\varphi \Delta \psi$ holds if and only if $\operatorname{Dom}(\psi) \subseteq \operatorname{Dom}(\varphi)$.

2. The identity $\varphi \Delta \psi=\varphi$ holds if and only if $\operatorname{Dom}(\psi) \cap \operatorname{Ran}(\varphi)=\varnothing$.

3. The identity $(\psi \Delta \varphi) \Delta \sigma=\psi \Delta(\varphi \Delta \sigma)$ holds if $\operatorname{Dom}(\sigma) \subseteq \operatorname{Dom}(\varphi)$ and $\sigma, \varphi, \psi$ do not contain variable renamings. 
The substitution join is not commutative but it is associative. Other properties are contained in the following lemma.

Lemma 4.6. Let $\varphi, \psi$ and $\sigma$ be substitutions.

1. The identity $(\varphi \Delta \psi) \sqcup \psi=\varphi \psi$ holds for every substitution $\varphi$ and $\psi$.

2. The identity $\varphi \sqcup \psi=\varphi$ holds if and only if $\operatorname{Dom}(\psi) \subseteq \operatorname{Dom}(\varphi)$.

3. The identity $\varphi \psi=\varphi \sqcup \psi$ holds if and only if $\operatorname{Dom}(\psi) \cap \operatorname{Ran}(\varphi)=\varnothing$.

The following lemma introduces a sufficient condition which assures the commutativity of the substitution join.

Lemma 4.7. Let $\varphi$ and $\psi$ be substitutions. The identity $\psi \sqcup \varphi=\varphi \sqcup \psi$ holds if $\operatorname{Dom}(\varphi) \cap \operatorname{Dom}(\psi)=\varnothing$.

The substitution join allows one to prove specific identity frequently used in the sequel.

Lemma 4.8. Let $\varphi$ and $\psi$ be substitutions. The identity $\psi(\varphi \Delta \psi)=\varphi \psi$ holds if $\operatorname{Dom}(\varphi) \cap \operatorname{Var}(\psi)=\varnothing$.

Proof. We have $\varphi \psi=(\varphi \Delta \psi) \sqcup \psi$ from Lemma 4.6, $(\varphi \Delta \psi) \sqcup \psi=\psi \sqcup$ $(\varphi \Delta \psi)$ from Lemma 4.7, $\psi \sqcup(\varphi \Delta \psi)=\psi(\varphi \Delta \psi)$ from Lemma 4.6 and $\operatorname{Dom}(\varphi \Delta \psi) \subseteq \operatorname{Dom}(\varphi)$.

The following property presents only a shorthand form of a long notation.

Definition 4.9. The substitutions $\varphi$ and $\psi$ are coherent (denote it by $\varphi \perp \psi$ ) if $\operatorname{Dom}(\varphi) \cap \operatorname{Var}(\psi)=\varnothing$ or $\operatorname{Var}(\varphi) \cap \operatorname{Dom}(\psi)=\varnothing$.

The coherence relation is symmetric.

There is no claim that the propositions presented entirely describe the properties of the defined operations. They represent only a working set necessary for proving properties of the objects in the following sections.

\subsection{Substitution Operators}

On the basis of substitution sum we define three iterative substitution operators - the exponent, W-operator and T-operator. For convenience and to remember their names, we can call the last two Whale and Turtle. Applying these iterative operators on a basic level substitution yields a graded sequence of new derived substitutions. The first two operators are necessary for closure chain definitions, the third one will be used in the crossed rule generalisation.

Definition 4.10. Let $\varphi$ and $\psi$ be substitutions. We define the iterative exponent operator on a substitution $\varphi$ inductively:

1. $\varphi^{0}=[]$

2. $\varphi^{n+1}=\varphi^{n} \varphi$

There is a generalisation of the Lemma 4.8 for the exponent operator.

Lemma 4.11. Let $\varphi$ and $\psi$ be substitutions. If $\operatorname{Dom}(\varphi) \cap \operatorname{Var}(\psi)=\varnothing$ then $\psi^{n}\left(\varphi \Delta \psi^{n}\right)=\varphi \psi^{n}$ holds for all $n$.

Proof. By induction on $n$ from Lemma 4.8 and $\operatorname{Dom}(\varphi) \cap \operatorname{Var}\left(\psi^{n}\right) \subseteq$ $\operatorname{Dom}(\varphi) \cap \operatorname{Var}(\psi)=\varnothing$. 
The W-operator represents a structural generalisation of the exponent operator.

Definition 4.12. Let $\varphi$ and $\psi$ be substitutions. We define the iterative operator $W$ (Whale) on $\varphi$ and $\psi$ inductively:

1. $W_{0}(\varphi, \psi)=[]$

2. $W_{n+1}(\varphi, \psi)=\left(\varphi \Delta W_{n}(\varphi, \psi)\right) \Delta \psi$

Because of their close relation, it would be interesting to know when the exponent and the W-operator coincide.

Lemma 4.13. Let $\varphi$ and $\psi$ be substitutions. If $\operatorname{Dom}(\psi) \cap \operatorname{Ran}(\varphi)=\varnothing$ then $W_{n}(\varphi, \psi)=\varphi^{n}$ hold for all $n$.

Proof. By induction on $n$.

Last but not least, we introduce the T-operator. This operator will not be used in closure chains but in the definition of crossed systems [Her89]. We present here only its definition and determine its connection to the previous operators.

Definition 4.14. Let $\varphi, \psi$ and $\sigma$ be substitutions. We define the iterative operator T (Turtle) on them inductively:

1. $T_{0}(\sigma, \psi, \varphi)=\sigma \Delta \varphi$

2. $T_{n+1}(\sigma, \psi, \varphi)=\left(\psi \Delta T_{n}(\sigma, \psi, \varphi)\right) \Delta \varphi$

The T-operator can be regarded as a slight extension of the W-operator. Because of their close relation it would be interesting to know when they coincide.

Fact 4.15. The identity $T_{n}(\psi, \psi, \varphi)=W_{n+1}(\psi, \varphi)$ holds for all $n$.

Proof. By induction on $n$.

The following proposition determines the connection between the $T$ - and exponent operators when the substitution $\sigma$ sifts upward.

Lemma 4.16. Let $\varphi, \psi$ and $\sigma$ be substitutions. If $\operatorname{Dom}(\varphi) \cap(\operatorname{Ran}(\psi) \cup$ $\operatorname{Ran}(\sigma))=\varnothing$ and $\operatorname{Dom}(\sigma) \subseteq \operatorname{Dom}(\psi)$, then $T_{n}(\sigma, \psi, \varphi)=\psi^{n} \Delta \sigma$ holds for all $n$.

Proof. By induction on $n$.

\section{Properties of Rule Closures}

In the first part we investigate the structure and construction of rule closures in general. The second part introduces special rule closures, called closure chains, together with the investigation of their properties.

\subsection{Structure and Construction of Rule Closures}

As can be easily seen from Definition 3.3, a duality principle determines the construction of forward and backward closures. 
Proposition 5.17 (Duality Principle). Let $R$ be a variable preserving rewrite system. Then $s \bowtie t$ is a forward closure in $F C(R)$ if and only if $t \mapsto s$ is a backward closure in $B C\left(R^{o p}\right)$.

According to this duality principle we do not need to distinguish between forward and backward closures in general but only in particular cases. If a result is provable for one kind of rule closures then it is provable also for the other kind according to this duality principle. Therefore, it we speak about rule closures we mean either forward or backward ones without further distinction. A rule closure without distinction will be denoted by $s \bowtie t$ which means either $s \nrightarrow t$ or $t \mapsto s$. A closure set of $R$ without distinction will be denoted by $R C(R)$ which means either $F C(R)$ or $B C\left(R^{o p}\right)$.

Let us investigate the construction of rule closures in particular. First, we introduce the closure operations according to Definition 3.3.

Definition 5.18. Let $R$ be an arbitrary set of rules. Let $p_{1}=s_{1} \triangleright t_{1}$, $p_{2}=s_{2} \mapsto t_{2}$ be two rule closures in $R C(R)$ such that $\left.t_{1}\right|_{a} \sigma=s_{2} \sigma$ holds with a most general unifier $\sigma$ and a non-variable position $a \in \mathscr{F} P o s\left(t_{1}\right)$. Then the rule closure $s_{1} \sigma \triangleright t_{1} \sigma\left[t_{2} \sigma\right]_{a}$ is constructible and the operation producing it is denoted by $p_{1} \leadsto a p_{2}$.

The notation $p \leadsto q$ is shorthand for $p \rightsquigarrow_{a} q$ for some $a$ understandable from the context.

Definition 3.3 might suggest a brute force procedure for producing the closure set $F C(R)$ or $B C(R)$ from $R$ based on the superposition of all newly produced rule closures to the existing ones. This can result in a rather inefficient way of producing the closure set (if it is finite) or deciding that the produced closure set is infinite. The following lemma suggests a more efficient procedure.

Lemma 5.19. Let $p_{1}, p_{2}$ and $p_{3}$ be rule closures of uniform kind. If the rule closure $q=p_{1} \varkappa_{a}\left(p_{2} \wedge_{b} p_{3}\right)$ is constructible then $q=\left(p_{1} \wedge_{a} p_{2}\right) \rightsquigarrow_{a b} p_{3}$.

Proof. Let $p_{1}=s_{1} \bowtie t_{1}, p_{2}=s_{2} \bowtie t_{2}$ and $p_{3}=s_{3} \bowtie t_{3}$ be the assumed rule closures. Let $\varphi$ be the substitution producing $p_{2} \wedge_{b} p_{3}$, i.e. $\left.t_{2}\right|_{b} \varphi=s_{3} \varphi$. Then

$$
p_{2} \gg_{b} p_{3}=s_{2} \varphi \triangleright t_{2} \varphi\left[t_{3} \varphi\right]_{b}
$$

is the resulting rule closure. Let $\psi$ be the substitution producing $p_{1} \varkappa_{a}$ $\left(p_{2} \wedge_{b} p_{3}\right)$, i.e.

$$
\left.t_{1}\right|_{a} \psi=s_{2} \varphi \psi
$$

Then

$$
p_{1} \rightsquigarrow_{a}\left(p_{2} \rightsquigarrow_{b} p_{3}\right)=s_{1} \psi \bowtie t_{1} \psi\left[t_{2} \varphi \psi\right]_{a}\left[t_{3} \varphi \psi\right]_{a b}
$$

is the constructed closure $q$.

The terms $\left.t_{1}\right|_{a}$ and $s_{2}$ are unifiable as shown by (6). Therefore the rule closure $q^{\prime}=p_{1} \leftrightarrow_{a} p_{2}$ is constructible. Let $\sigma$ be the most general unifier of $\left.t_{1}\right|_{a}$ and $s_{2}$ involved in the construction of $q^{\prime}$. From the definition of a most general unifier and from (6) follows that there exists a substitution $\rho$ such that $\psi \cup(\varphi \psi)=\sigma \rho$. Comparing these substitutions with (7) implies that the rule closure

$$
\left(p_{1} \sim_{a} p_{2}\right) \sim_{a b} p_{3}
$$

is constructible and is equal to the rule closure $q$. 
The previous lemma proves a semi-associativity of the closure operations. Forward closures are left-, backward ones are right-associative. Therefore the parentheses in closure constructions can be omitted.

Corollary 5.20. Let $R$ be a variable preserving rewrite system. If $p$ is a rule closure from $R C(R)-R$, respectively $R C(R)-R^{o p}$, then it can be written as a composition $p=q_{1} \leadsto q_{z}$ where $q_{1} \in R C(R)$ and $q_{2}$ is a rewrite rule in $R$, respectively $R^{o p}$.

Proof. By induction on the number of the closure operations $m$, with the support of Lemma 5.19.

From this corollary, it follows that a new rule closure can be produced from an already existing one and a basic rewrite rule. This is the principle of the closure producing Procedure 5.21. This procedure is the same for both forward and backward closure sets, except for the closure producing operation. Therefore, we present only one parametric procedure instead of two particular ones. The parameter to be supplied is the particular closure operation.

Procedure 5.21 (Closure Set Generation)

Input: $R$, respectively $R^{\text {op }}$

Output: $R C(R)$ if it halts

Method:

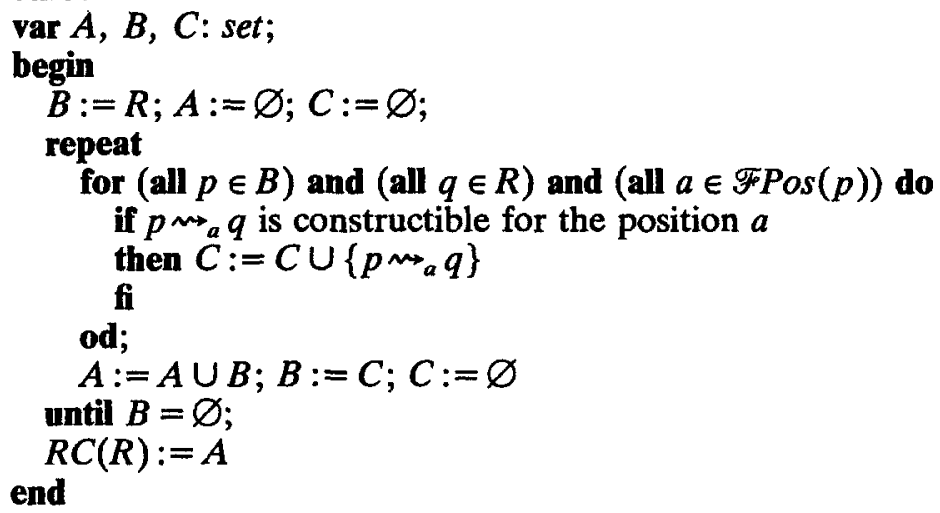

Alas, this procedure is even more prone to produce an infinite (closure) set than the classical Knuth-Bendix procedure, as it was defined by Huet [Hue81]. Even the fact that the closure set $R C(R)$ is (in)finite is undecidable for an arbitrary rewrite system $R$. This proposition can be proved by a modification of the termination undecidability proof.

Theorem 5.22. It is undecidable if the closure set $R C(R)$ of a rewrite system $R$ is finite, even if $R$ has only two rules, one of them a ground rule containing no variables.

Proof. As proved by Dauchet [Dau89]. Turing machines can be simulated by single-rule left-linear variable preserving rewrite systems. Let $R^{\prime}$ be such a rewrite system. Now, the rewrite system $R$ is constructed from $R^{\prime}$ by addition of a new ground rule $s \rightarrow t$. The rewriting process of the term $t$ by the system $R^{\prime}$ can be transformed to a forward closure generating process from the rule $s \rightarrow t$ in the system $R$ only by formal changes. Instead of generating the rewriting sequence $t \rightarrow_{R^{\prime}} t_{1} \rightarrow_{R^{\prime}} t_{2} \rightarrow_{r^{\prime}} \cdots$ one has to generate the forward 
closure sequence $s \bowtie t, s \bowtie t_{1}, s \mapsto t_{2} \cdots$ by the same means. This reasoning can be extended to backward closures using the Duality Principle 5.17. The decidability problem of the closure set finiteness can be reduced now to the decidability problem of the (weak) termination of rewrite systems.

As proved by Dauchet in the above mentioned paper [Dau89], (weak) termination is undecidable even for single-rule left-linear variable preserving rewrite systems.

Even if only restricted systems are taken into account, the undecidability property remains valid.

Corollary 5.23. It is undecidable if the closure set $R C(R)$ of a rewrite system $R$ is finite, even if it is non-overlapping and contains only monadic function symbols and constants.

Proof. Huet and Lankford [HuL78] proved the termination undecidability theorem under the restriction to monadic function symbols and constants in non-overlapping systems. Moreover, the system constructed in their proof is variable preserving. Applying to their proposition the same argument as in the proof of the previous theorem gives the desired result.

\subsection{Closure Chains}

We define a special kind of rule closures called closure chains. We illustrate these closure chains by some particular but sufficiently general examples. As in the case of rule closures, two types of closure chains basically appear - forward and backward chains. Close similarity between the construction of these two kinds of chains can be observed. Afterwards we give characterisation theorems for closure sets when they contain a chain, showing that this property is sufficient for the infinity of $R C(R)$. Actually, our interest is focused on the closure chains, because according to their construction they are responsible for the divergence of Knuth-Bendix completion procedure [Her89].

Section 5.1 described the structure and construction of rule closures $R C(R)$. Now we introduce a special but sufficiently general class of rule closures, whose appearance is essential for generation of an infinite closure set in their own right. Proving the properties of closure chains has been made possible by the small substitution algebra developed in Section 4. Also the principle of substitutions in own variables becomes clear from the following general explanation.

Let us consider a rule closure

$$
s \triangleright t
$$

with the requirement that it should be possible to chain it with itself. This means that the term $s$ must be unifiable with a non-variable subterm $\left.t\right|_{b}$. Unification is possible only if the unified terms have disjoint variables, which is not the case here. Therefore we need to perform variable renaming. If we want to control the chaining iteration, variable renaming must be performed explicitly. These considerations lead to the split of a unifier into variable renamings and substitutions in own variables.

First, we need a variable renaming $\rho_{0}=\left[x \mapsto x_{0} \mid x \in \operatorname{Var}(s)\right]$ to rename the variables in (8) by introducing indexes to them. Now, we can consider the 
chaining of the rule closures $s \rho_{0} \bowtie t \rho_{0}=s_{0} \bowtie t_{0}$ and $s \bowtie t$. The actual most general unifier in the superposition of previous rule closures will be split into the substitutions $\varphi_{1}$ and $\varphi_{2}$ in own variables of $s$, such that

$$
\left.t\right|_{b} \varphi_{1}=s \varphi_{2}
$$

holds, and the variable renaming $\pi_{0}=\left[x_{0} \mapsto x \mid x_{0} \in \operatorname{Var}\left(\left.t_{0}\right|_{b}\right)\right]$ cancelling some of the indices introduced by $\rho_{0}$, to allow the application of the substitution $\varphi_{1}$ to the subterm $\left.t\right|_{b}$. To assure the idempotence of the unifier, we apply the variable renaming $\rho_{1}=\left[x \mapsto x_{1} \mid x \in \operatorname{Var}(s)\right]$ introducing new indices at the end. Thus, the actual unifier will be $\sigma=\left(\left(\pi_{0} \Delta \varphi_{1}\right) \cup \varphi_{2}\right) \rho_{1}$ and the rule closure yielded by the first iteration step is

$$
s_{0}\left(\pi_{0} \Delta \varphi_{1}\right) \rho_{1} \triangleright t_{0}\left(\pi_{0} \Delta \varphi_{1}\right) \rho_{1}\left[t \varphi_{2} \rho_{1}\right]_{b}
$$

Up to this step there is nothing interesting in this process, except that variable renaming is performed explicitly. Let us suppose further that the newly created rule closure (10) and the previous rule closure (8) build another rule closure, and that this process may be continued indefinitely: the rule closure, produced during the $n$th iteration step, and the rule closure (8) always build a new rule closure.

Let us thini of the rule closure (10) in the form $s_{1} \bowtie t_{1}$. According to the previous assumption, in the second iteration there must be the substitutions $\varphi_{1}^{\prime}, \varphi_{2}^{\prime}$ in own variables of $s$, the position $b^{\prime} \in \mathscr{F} P o s\left(t_{1}\right)$, and the variable renaming $\pi_{1}=\left[x_{1} \mapsto x \mid x_{1} \in \operatorname{Var}\left(\left.t_{1}^{\prime}\right|_{b}\right)\right]$, such that

$$
\left.t_{1}^{\prime}\right|_{b}\left(\pi_{1} \Delta \varphi_{1}^{\prime}\right)=s \varphi_{2}^{\prime}
$$

holds. Of course, this is only a recalling of condition (9) from the first iteration. It is natural to think of $b^{\prime}$ as an iteration of $b$, i.e. $b^{\prime}=b \cdot b$, because this position exists in $t_{1}$ in each particular case, although in general $\left.t\right|_{b}$ is not the only subterm of $t$ which can unify with $s$. This means that (11) can be transformed to

$$
\left.t\right|_{b} \varphi_{2} \varphi_{1}^{\prime}=s \varphi_{2}^{\prime}
$$

The substitutions $\varphi_{1}^{\prime}$ and $\varphi_{2}^{\prime}$ will certainly not be completely new but it is natural to think of them as functions of the previous substitutions $\varphi_{1}$ and $\varphi_{2}$. Therefore we introduce the notion of a particular substitution function, in this case

$$
\begin{aligned}
& \varphi_{1}^{\prime}=f_{1}\left(\varphi_{1}, \varphi_{2}\right) \\
& \varphi_{2}^{\prime}=f_{2}\left(\varphi_{1}, \varphi_{2}\right)
\end{aligned}
$$

Let us now compare identities (9) and (12). Of course identity (9) can be extended to

$$
\left.t\right|_{b} \varphi_{1} \psi^{\prime}=s \varphi_{2} \psi^{\prime}
$$

for all substitutions $\psi^{\prime}$ according to the composition properties, but such an extension does not give appropriate results and does not suit our goals. We must proceed cleverly and extend the identity (9) to a more general one

$$
t_{b}\left(\varphi_{1} \Delta \psi^{\prime}\right)=s\left(\varphi_{2} \Delta \psi^{\prime}\right)
$$

It is also natural to consider the new extending substitution $\psi^{\prime}$ in terms of $\varphi_{1}$ 
and $\varphi_{2}$, introducing a third substitution function

$$
\psi^{\prime}=f_{3}\left(\varphi_{1}, \varphi_{2}\right)
$$

Comparing identities (12) and (15) in terms of the defined substitution functions $f_{1}, f_{2}$ and $f_{3}$ we get the following identities

$$
\begin{aligned}
\varphi_{2} f_{1}\left(\varphi_{1}, \varphi_{2}\right) & =\varphi_{1} \dot{\Delta} f_{3}\left(\varphi_{1}, \varphi_{2}\right) \\
f_{2}\left(\varphi_{1}, \varphi_{2}\right) & =\varphi_{2} \Delta f_{3}\left(\varphi_{1}, \varphi_{2}\right)
\end{aligned}
$$

which express the conditions that must be satisfied in the second iteration step.

As described earlier, we require that it must be possible to iterate this process indefinitely. This means that an iterated infinite rule closure sequence $s_{n} \nrightarrow t_{n}$, where $s_{n} \triangleright t_{n}=\left(s_{n-1} \hookleftarrow t_{n-1}\right) \leadsto b^{n}(s \triangleright t)$, must be constructible from $s \bowtie t$, by means of the iterated substitution sequences ${ }^{3}$

$$
\begin{aligned}
& \varphi_{1}^{(n)}=f_{1}^{(n)}\left(\varphi_{1}, \varphi_{2}\right) \\
& \varphi_{2}^{(n)}=f_{2}^{(n)}\left(\varphi_{1}, \varphi_{2}\right) \\
& \psi^{(n)}=f_{3}^{(n)}\left(\varphi_{1}, \varphi_{2}\right)
\end{aligned}
$$

taking advantage of the explicit variable renaming by a pair of fold/unfold substitutions

$$
\begin{aligned}
& \pi_{n}=\left[x_{n} \mapsto x \mid x_{n} \in \operatorname{Var}\left(\left.t_{n}\right|_{b^{n+1}}\right)\right] \\
& \rho_{n}=\left[x \mapsto x_{n} \mid x \in \operatorname{Var}(s)\right]
\end{aligned}
$$

for each iteration step $n$, which cancel and introduce indices. The actual unifier in the iteration step $n$ will be constructed from $\varphi_{1}^{(n)}, \varphi_{2}^{(n)}, \pi_{n}$, and $\rho_{n+1}$ as

$$
\sigma_{n}=\left(\left(\pi_{n} \Delta \varphi_{1}^{(n)}\right) \cup \varphi_{2}^{(n)}\right) \rho_{n+1}
$$

The iterated substitution sequences $\varphi_{1}^{(n)}, \varphi_{2}^{(n)}$ and $\psi^{(n)}$ are derived from the notion of substitution function, introduced by the identities (13), (14) and (16). Therefore the new substitution functions $f_{1}^{(n)}, f_{2}^{(n)}$ and $f_{3}^{(n)}$ are built up as iterations of the basic ones $f_{1}, f_{2}$ and $f_{3}$. Conditions (17) and (18) are to be extended in a similar manner.

As it is to be shown in the following propositions, the previously described iterations are granted by the substitution algebra constructions, especially by the iterative substitution operators exponent and Whale, developed in Section 4. Hence the closure chain definitions inherit the conditions of their supporting substitution algebra constructions. Particularly, these are the conditions from Lemma 4.8, propagated through Lemma 4.11. Last but not least, the rule closure (8) can be enlarged to a mixed structure - to an overlap closure.

Definition 5.24. An overlap closure $s \leftrightarrow t$ is a forward chain if there are substitutions $\varphi_{1}, \varphi_{2}$ in own variables of $s$ and position $b \in \mathscr{F} P o s(t)$ such that

1. $\left.t\right|_{b} \varphi_{1}=s \varphi_{2}$

2. $\varphi_{1} \perp \varphi_{2}$

Example 5.25. There exist two cases of forward chains depending on the choice of the second condition in the coherence relation.

\footnotetext{
${ }^{3}$ We have $\varphi_{i}^{(1)}=\varphi_{r}^{\prime}$ and $f_{i}^{(1)}=f_{r}$.
} 
1. Consider the forward closure $(x \oplus y) \otimes y \bowtie k(x \otimes k(y))$. There are substitutions $\varphi_{1}=[x \mapsto x \oplus k(y)], \varphi_{2}=[y \mapsto k(y)]$ and the position $b=1$ satisfying $\left.t\right|_{b} \varphi_{1}=s \varphi_{2}$ and $\operatorname{Dom}\left(\varphi_{1}\right) \cap \operatorname{Var}\left(\varphi_{2}\right)=\varnothing$. Therefore this forward closure belongs to the first kind of forward chain.

2. Consider the forward closure $g(x) \oplus y \triangleright g(x \oplus(x \oslash y))$. There are substitutions $\varphi_{1}=[x \mapsto g(x)], \varphi_{2}=[y \mapsto g(x) \oslash y]$ and the position $b=1$ satisfying $\left.t\right|_{b} \varphi_{1}=s \varphi_{2}$ and $\operatorname{Dom}\left(\varphi_{2}\right) \cap \operatorname{Var}\left(\varphi_{1}\right)=\varnothing$. This forward closure belongs to the second kind of forward chain.

Someone may argue that there exist overlap closures which do not satisfy the coherence condition and, nevertheless, build on an infinite iteration sequence, as e.g. the forward closure $f(x \oplus y, y, u, x \otimes z) \longmapsto f(g(g(y)) \oplus$ $x, g(y), h(u), z)$. It can be proved that the coherence relation becomes satisfied after the first iteration step.

Backward chains represent the counterpart of the forward chains. They are constructed from backward closures in a similar way. Also a certain kind of duality associated with forward and backward chains can be observed.

Definition 5.26. An overlap closure $s \leftrightarrow t$ is a backward chain if there are substitutions $\varphi_{1}, \varphi_{2}$ in own variables of $s$ and position $b \in \mathscr{F} P o s(s)$ such that

1. $t \varphi_{1}=\left.s\right|_{b} \varphi_{2}$

2. $\varphi_{1} \perp \varphi_{2}$

Example 5.27. There exist two cases of backward chains depending on the choice of the second condition in the coherence relation.

1. Consider the backward closure $d(x \oplus(x \otimes y)) \mapsto d(x) \oplus y$. There are substitutions $\varphi_{1}=[y \mapsto d(x) \otimes y], \varphi_{2}=[x \mapsto d(x)]$ and the position $b=1$ satisfying $t \varphi_{1}=\left.s\right|_{b} \varphi_{2}$ and $\operatorname{Dom}\left(\varphi_{1}\right) \cap \operatorname{Var}\left(\varphi_{2}\right)=\varnothing$. Therefore this backward closure belongs to the first kind of backward chain.

2. Consider the backward closure $(x \otimes h(y)) \oplus y \rightarrow(x \oplus y) \otimes y$. There are substitutions $\varphi_{1}=[y \mapsto h(y)], \varphi_{2}=[x \mapsto x \oplus h(y)]$ and the position $b=1$ satisfying $t \varphi_{1}=\left.s\right|_{b} \varphi_{2}$ and $\operatorname{Dom}\left(\varphi_{2}\right) \cap \operatorname{Var}\left(\varphi_{1}\right)=\varnothing$. This backward closure belongs to the second kind of backward chain.

The same discussion as for the forward chain, concerning the coherence conditions and chaining property, appears also for the backward chain.

Example 5.28. Of course, not every rule closure builds a closure chain. Consider the rewrite system

$$
\begin{aligned}
x \otimes k(y) & \rightarrow k(x \oplus k(y)) \\
(x \oslash y) \oplus y & \rightarrow g(x) \otimes y
\end{aligned}
$$

Its forward closure set contains these rewrite rules transformed to closures and the following composed forward closures

$$
\begin{aligned}
& (x \oslash k(y)) \otimes k(y) \triangleright k(g(x) \otimes k(y)) \\
& (x \oslash k(y)) \oplus k(y) \triangleright k(g(x) \oplus k(y)) \\
& (x \oslash k(y)) \otimes k(y) \bowtie k^{2}(g(x) \oplus k(y))
\end{aligned}
$$

There is no forward chain among them and the closure set is finite. As we will prove later, the finiteness of the closure set requires the nonexistence of a forward chain. 
The next theorem describes closure sets by means of closure chains. Actually, these are two theorems, one for the forward chain, the other for the backward one. The proof for closure chains corresponds syntactically to that for forward chains. The result for backward chains can be proved in a similar manner. The same argument applies to all following results in this section.

Theorem 5.29. If $s \bowtie t$ is a closure chain, where $s \neq t$, then $R C(\{s \rightarrow t\})$ is infinite.

Proof. Let $s \bowtie t$ be a closure chain as in Definition 5.24 or 5.26. Let

$$
\begin{aligned}
s_{0} & =s \rho_{0} \\
s_{n+1} & =s_{n}\left(\pi_{n} \Delta\left(\varphi_{1} \Delta W_{n}\left(\varphi_{2}, \varphi_{1}\right)\right)\right) \rho_{n+1} \\
t_{0} & =t \rho_{0} \\
t_{n+1} & =t_{n}\left(\pi_{n} \Delta\left(\varphi_{1} \Delta W_{n}\left(\varphi_{2}, \varphi_{1}\right)\right)\right) \rho_{n+1}\left[t\left(\varphi_{2} \Delta W_{n}\left(\varphi_{2}, \varphi_{1}\right)\right) \rho_{n+1}\right]_{b^{n+1}}
\end{aligned}
$$

define a sequence of terms $s_{n}$ and $t_{n}$. We will prove that $s_{n} \triangleright t_{n}$ is in $R C(\{s \rightarrow t\})$ for all $n$ by induction.

Base: $n=0$. The rule closure $s_{0} \bowtie t_{0}$ is actually a substitution instance of $s \triangleright t$.

Step: Let the proposition hold for $n \leq k$. We will prove it for $k+1$.

The rule closures $s_{k} \triangleright t_{k}$ and $s \bowtie t$ are in $R C(\{s \rightarrow t\})$. We will construct the rule closure $s_{k+1} \bowtie t_{k+1}$ from them. We must analyse two possibilities according to the definitions of closure chains.

1. $\operatorname{Dom}\left(\varphi_{1}\right) \cap \operatorname{Var}\left(\varphi_{2}\right)=\varnothing$.

In this case we have $\varphi_{1} \Delta W_{n}\left(\varphi_{2}, \varphi_{1}\right)=\varphi_{1} \Delta \varphi_{2}^{n}$ and $\varphi_{2} \Delta W_{n}\left(\varphi_{2}, \varphi_{1}\right)=$ $\varphi_{2}^{n+1}$ from Lemma 4.13. So we get

$$
\begin{aligned}
& s_{n+1}=s_{n}\left(\pi_{n} \Delta\left(\varphi_{1} \Delta \varphi_{2}^{n}\right)\right) \rho_{n+1} \\
& t_{n+1}=t_{n}\left(\pi_{n} \Delta\left(\varphi_{1} \Delta \varphi_{2}^{n}\right)\right) \rho_{n+1}\left[t \varphi_{2}^{n+1} \rho_{n+1}\right]_{b^{n+1}}
\end{aligned}
$$

From this we have $\left.t_{k}\right|_{b^{k+1}} \pi_{k}=\left.t\right|_{b} \varphi_{2}^{k}$ for $k \geq 1$, and from Lemma 4.11 it is possible to prove $\left.t\right|_{b} \varphi_{2}^{k}\left(\varphi_{1} \triangle \varphi_{2}^{k}\right)=s \varphi_{2}^{k+1}$. From this identity if follows that $\left.t_{k}\right|_{b^{k+1}}\left(\pi_{k} \Delta\left(\varphi_{1} \Delta \varphi_{2}^{k}\right)\right) \rho_{k+1}=s \varphi_{2}^{k+1} \rho_{k+1}$ which satisfies the definition of rule closure with the unifier $\left(\left(\pi_{k} \Delta\left(\varphi_{1} \Delta \varphi_{2}^{k}\right)\right) \cup \varphi_{2}^{k+1}\right) \rho_{k+1}$. Then the construction

$$
s_{k}\left(\pi_{k} \Delta\left(\varphi_{1} \Delta \varphi_{2}^{k}\right)\right) \rho_{k+1} \bowtie t_{k}\left(\pi_{k} \Delta\left(\varphi_{1} \Delta \varphi_{2}^{k}\right)\right) \rho_{k+1}\left[t \varphi_{2}^{k+1} \rho_{k+1}\right]_{b^{k+1}}
$$

is also a rule closure in $R C(\{s \rightarrow t\})$, and this is exactly $s_{k+1} \triangleright t_{k+1}$.

2. $\operatorname{Dom}\left(\varphi_{2}\right) \cap \operatorname{Var}\left(\varphi_{1}\right)=\varnothing$.

In this case we have $\varphi_{1} \Delta W_{n}\left(\varphi_{2}, \varphi_{1}\right)=\varphi_{1}$ according to Lemma 4.5 and $\operatorname{Dom}\left(W_{n}\left(\varphi_{2}, \varphi_{1}\right)\right) \subseteq \operatorname{Dom}\left(\varphi_{2}\right)$. So we get

$$
\begin{aligned}
& s_{n+1}=s_{n}\left(\pi_{n} \Delta \varphi_{1}\right) \rho_{n+1} \\
& t_{n+1}=t_{n}\left(\pi_{n} \Delta \varphi_{1}\right) \rho_{n+1}\left[t\left(\varphi_{2} \Delta W_{n}\left(\varphi_{2}, \varphi_{1}\right)\right) \rho_{n+1}\right]_{b^{n+1}}
\end{aligned}
$$

From this we have $\left.t_{k}\right|_{b^{k+1}} \pi_{k}=\left.t\right|_{b}\left(\varphi_{2} \Delta W_{k-1}\left(\varphi_{2}, \varphi_{1}\right)\right)$ for $k \geq 1$, and from Lemma 4.8 it is possible to prove $\left.t\right|_{b}\left(\varphi_{2} \Delta W_{k-1}\left(\varphi_{2}, \varphi_{1}\right)\right) \varphi_{1}=$ $s\left(\varphi_{2} \Delta W_{k}\left(\varphi_{2}, \varphi_{1}\right)\right)$. From this identity it follows that $\left.t_{k}\right|_{b^{k+1}}\left(\pi_{k} \Delta \varphi_{1}\right) \rho_{k+1}=s\left(\varphi_{2} \Delta W_{k}\left(\varphi_{2}, \varphi_{1}\right)\right) \rho_{k+1}$ which satisfies the definition of rule closure with the unifier $\left(\left(\pi_{k} \Delta \varphi_{1}\right) \cup\left(\varphi_{2} \Delta W_{k}\left(\varphi_{2}, \varphi_{1}\right)\right)\right) \rho_{k+1}$. 
Then the construction

$$
s_{k}\left(\pi_{k} \Delta \varphi\right) \rho_{k+1} \triangleright t_{k}\left(\pi_{k} \Delta \varphi_{1}\right) \rho_{k+1}\left[t\left(\varphi_{2} \Delta W_{k}\left(\varphi_{2}, \varphi_{1}\right)\right) \rho_{k+1}\right]_{b^{k+1}}
$$

is also a rule closure in $R C(\{s \rightarrow t\})$, and this is exactly $s_{k+1} \triangleright t_{k+1}$.

Let us describe the rule closures $s \bowtie s$ with the same terms on both sides as reflexive.

Corollary 5.30. If $R C(R)$ contains a non-reflexive closure chain then $R C(R)$ is infinite.

The following corollary proves that the test for determining a closure chain can be performed on every step of the closure set generating procedure.

Corollary 5.31. Each rule closure $s_{n} \triangleright \triangleright t_{n}$ from the proof of the Theorem 5.29 is a closure chain.

Proof. The existence of the substitutions for the first condition of closure chains is a consequence of the proof of Theorem 5.29, combined with the restricted right associativity of the closure construction, presented in the following propositon.

Let $p_{1}, p_{2}$ and $p_{3}$ be rule closures of uniform kind. If the rule closure $q=\left(p_{1} \rightarrow_{a} p_{2}\right) \sim_{a b} p_{3}$ is constructible and $b \in \mathscr{F} P o s\left(t_{2}\right)$, where $p_{2}=$ $s_{2} \bowtie t_{2}$, then $q=p_{1} \varkappa_{a}\left(p_{2} \aleph_{b} p_{3}\right)$.

The proof of this proposition can be found in the appendix of [GKM83].

The rest consists of proving the coherence property for the generated closure sequence. We will actually prove that the required coherence conditions hold in each basic step between the constructed closure chain iteration and the starting chain.

For the proof of the coherence property let us consider the replacing substitutions $\varphi_{1} \Delta W_{n}\left(\varphi_{2}, \varphi_{1}\right)$ and $\varphi_{2} \Delta W_{n}\left(\varphi_{2}, \varphi_{1}\right)$ instead of $\varphi_{1}$ and $\varphi_{2}$. We must analyse two possibilities as has been done in the proof of Theorem 5.29.

1. $\operatorname{Dom}\left(\varphi_{1}\right) \cap \operatorname{Var}\left(\varphi_{2}\right)=\varnothing$.

From the substitution sum and exponent operator definitions it follows that

$$
\begin{aligned}
\operatorname{Dom}\left(\varphi_{1} \triangle \varphi_{2}^{n}\right) & \subseteq \operatorname{Dom}\left(\varphi_{1}\right) \\
\operatorname{Var}\left(\varphi_{2}^{n}\right) & \subseteq \operatorname{Var}\left(\varphi_{2}\right)
\end{aligned}
$$

Substituting the new constructed substitutions $\varphi_{1} \Delta \varphi_{2}^{n}$ and $\varphi_{2}^{n+1}$ for $\varphi_{1}$ and $\varphi_{2}$ respectively into the first part of the second coherence condition produces the identity

$$
\operatorname{Dom}\left(\varphi_{1} \triangle \varphi_{2}^{n}\right) \cap \operatorname{Var}\left(\varphi_{2}^{n+1}\right) \subseteq \operatorname{Dom}\left(\varphi_{1}\right) \cap \operatorname{Var}\left(\varphi_{2}\right)=\varnothing
$$

2. $\operatorname{Dom}\left(\varphi_{2}\right) \cap \operatorname{Var}\left(\varphi_{1}\right)=\varnothing$.

Substituting the newly constructed substitution $\varphi_{2} \Delta W_{n}\left(\varphi_{2}, \varphi_{1}\right)$ for $\varphi_{2}$ into the first part of the second coherence condition produces the identity

$$
\operatorname{Dom}\left(\varphi_{2} \Delta W_{n}\left(\varphi_{2}, \varphi_{1}\right)\right) \cap \operatorname{Var}\left(\varphi_{1}\right) \subseteq \operatorname{Dom}\left(\varphi_{2}\right) \cap \operatorname{Var}\left(\varphi_{1}\right)=\varnothing
$$


Using Corollary 5.31, the closure chains in the closure set can be classified according to their initial construction.

Definition 5.32. A closure chain $c=s \longmapsto t$ from $R C(R)$ is independent in $R C(R)$ if there is no other closure chain $c^{\prime}=s^{\prime} \triangleright t^{\prime}$ in $R C(R)$, such that $c=s_{n}^{\prime} \triangleright t_{n}^{\prime}$ for some iteration step $n$ of the chain $c^{\prime}$.

The last problem to solve is whether it is decidable that a closure set $R C(R)$ contains a closure chain.

Theorem 5.33. It is undecidable in general whether $R C(R)$ contains a closure chain.

Proof. The proof consists of a simple modification of the undecidability proof of Narendran and Stillman [NaS89]. Only a sketch of the proof is presented here.

First, a deterministic Turing machine $M$ is coded by a nonoverlapping rewrite system $R_{M}$. Let $s \rightarrow t$ be a forward chain of symbols not contained in $R_{M}$. We split the chain into more rules. We construct the rule

$$
s \rightarrow s_{w}
$$

where $s_{w}$ is a term coding the starting instantaneous description of $M$ for an input word $w$. Further we construct the rule(s)

$$
t_{w} \rightarrow t
$$

for the final state(s), where $t_{w}$ is a term coding the accepting instantaneous description of $M$ for $w$. We take $R=R_{M} \cup\left\{s \rightarrow s_{w}\right\} \cup\left\{t_{w} \rightarrow t\right\}$. By the same method as in [NaS89] it can be shown that computing the forward closures starting from (19) in $F C(R)$ simulates the computation of the machine $M$. The closure set $F C(R)$ contains the forward chain $s \rightarrow t$ if and only if the Turing machine $M$ halts and accepts the input word $w$.

The halting problem for Turing machines is reduced to the problem of generating a forward chain. The same result for a backward chain can be proved in a similar manner.

\section{Conclusion}

First, we introduced a special substitution algebra with a connection to the divergence problem of completion. After that we studied some properties of rule closures in general, pointing out the undecidability of the closure set finiteness. Last but not least, we defined a special class of rule closures, called closure chains, identified then formally as the core reason of divergence, and proved their iterated generation by means of the previously introduced substitution algebra.

Because the closure chains were identified as the underlying notion for divergence, the determination of a system as divergent reduces to a search for closure chains. Unfortunately, it is undecidable in general to determine if a closure chain will be generated. 


\section{Acknowledgements}

I would like to thank Hélène Kirchner, Leo Bachmair, Laurent Fribourg, Pierre Lescanne, Igor Prívara, and Pierre Réty for comments and suggestions on the early versions of this paper, and Philip Feinsilver who helped me with the linguistic revision.

\section{References}

[BeL87] BenCherifa, A. and Lescanne, P.: Termination of Rewriting Systems by Polynomial Interpretations and Its Implementation. Science of Computer Programming, 9(2), 137-159 (1987).

[Dau89] Dauchet, M.: Simulation of Turing Machines by a Left-Linear Rewrite Rule. In: Proc. 3rd Conf. Rewriting Techniques and Applications, Chapel Hill, (North Carolina, USA), N. Dershowitz (ed.), pp. 109-120, Lecture Notes in Computer Science 355, Springer-Verlag, 1989.

[Der87] Dershowitz, N.: Termination of Rewriting. Journal of Symbolic Computation, 3(1 \& 2), 69-116 (1987). (Special issue on rewriting techniques and applications.)

[DeJ89] Dershowitz, N. and Jouannaud, J.-P.: Notations for Rewriting, unpublished note, October 1989.

[Fay79] Fay, M.: First-Order Unification in an Equational Theory. In: Proc. 4th Workshop on Automated Deduction, S. Sickel, (ed.), pp. 161-167, Austin, TX, February 1979.

[GKM83] Guttag, J. V., Kapur, D. and Musser, D. R.: On Proving Uniform Termination and Restricted Termination of Rewrite Systems. SIAM Journal on Computation, 12(1), 189-214 (1983).

[Her89] Hermann, M.: Crossed Term Rewriting Systems, Research report 89-R-003, Centre de Recherche en Informatique de Nancy, 1989. (Submitted for publication.)

[HeP86] Hermann, M. and Prívara, I.: On Nontermination of Knuth-Bendix Algorithm, In: Proceedings 13th ICALP Conference, Rennes (France), L. Kott, (ed.), pp. 146-156, Lecture Notes in Computer Science 226. Springer-Verlag, 1986.

[Hue81] Huet, G.: A Complete Proof of Correctness of the Knuth-Bendix Completion Algorithm. Journal of Computer and System Science. 23(1), 11-21 (1981). (Also as: Rapport 25. INRIA, 1980).

[HuL78] Huet, G. and Lankford, D. S.: On the Uniform Halting Problem for Term Rewriting Systems. Rapport de recherche 283, Institute de Recherche en Informatique et en Automatique, Le Chesnay, France, 1978.

[Hul80] Hullot, J.-M.: Canonical Forms and Unification. In: Proc. 5th Conf. on Automated Deduction, Les Arcs (France), W. Bibel and R. Kowalski (eds), Lecture Notes in Computer Science 87, pp. 318-334, Springer-Verlag, New York, 1980.

[Kir89] Kirchner, H.: Schematization of Infinite Sets of Rewrite Rules Generated by Divergent Completion Process. Theoretical Computer Science, 67(2-3), 303-332 (1989).

[Lan75] Lankford, D. S.: Canonical Inference, Research report ATP-32. Department of Mathematics and Computer Science, University of Texas, Austin, TX, December 1975.

[LaM78] Lankford, D. S. and Musser, D. R.: A Finite Termination Criterion, Unpublished draft, Information Sciences Institute, University of Southern California, Marina-delRey, CA, 1978.

[Les83] Lescanne, P.: Computer Experiments with the REVE Term Rewriting System Generator. In Proc. 10th ACM POPL Symp., Austin, TX, pp. 99-108, 1983.

[NaS89] Narendran, P. and Stillman, J.: It is Undecidable Whether the Knuth-Bendix Completion Procedure Generates a Crossed Pair. In Proc. 6th STACS, Paderborn (FRG), B. Monien and R. Cori, (eds), pp. 348-359. Lecture Notes in Computer Science, 349, Springer-Verlag, 1989.

[Ros73] Rosen, B. K.: Tree-Manipulating Systems and Church-Rosser Theorems. Journal of $A C M, 20(1), 160-187$ (1973).

[Sla74] Slagle, J. R.: Automated Theorem-Proving for Theories with Simplifiers, Commutativity, and Associativity. Journal of ACM, 21, 662-642 (1974). 


\section{Appendix. Index of Symbols}

\begin{tabular}{ll}
\hline Symbol & Meaning \\
\hdashline & Forward closure \\
$\mapsto$ & Backward closure \\
$\mapsto$ & Overlap closure \\
$\mapsto$ & Rule closure \\
$F C(-)$ & Rule closure constructor \\
$B C(-)$ & Forward closure set \\
$O C(-)$ & Backward closure set \\
$R C(-)$ & Overlap closure set \\
$\triangle$ & Rule closure set \\
$\sqcup$ & Substitution sum \\
$\perp$ & Substitution composition \\
$\perp$ & Substitution join \\
$\bar{W}^{n}$ & Coherence relation \\
$T_{n}(-,-)$ & Substitution operator (exponent) \\
$T_{n}(-,-,-)$ & Substitution operator (Whale) \\
\hline
\end{tabular}

Received January 1989

Accepted in a revised form January 1990 by U. H. M. Martin 